\title{
LAS REDES COMO FACTOR CLAVE PARA LA \\ CONSOLIDACIÓN DE NUEVAS COOPERATIVAS DE \\ TRABAJO ASOCIADO
}

\author{
POR \\ Rubén J. CUÑAT GIMÉNEZ
}

\section{RESUMEN}

En estos últimos años de crisis y destrucción de empleo, el interés por impulsar el dinamismo y crecimiento de la economía de un área geográfica a través de la creación de nuevas empresas ha ido en aumento. Las cooperativas de trabajo asociado (CTA) representan un modelo de empresa en el que los objetivos económicos se combinan con los sociales, logrando un crecimiento basado en el empleo, la igualdad social y la equidad.

Las nuevas CTA necesitan conocimiento para su consolidación, una parte del cual proviene de la formación y experiencia de sus promotores, y otra de las relaciones de tipo interpersonal e interorganizacional dentro de su entorno económico y social. La supervivencia y crecimiento de la CTA se verá muy influida por su habilidad para mantener y extender sus redes.

Este trabajo plantea como objetivo contribuir al conocimiento de los diferentes tipos de redes con los que se relaciona una cooperativa desde su nacimiento hasta la consolidación, así como el impacto que tienen en su supervivencia y crecimiento.

Para ello se han realizado un total de 36 entrevistas en profundidad utilizando una metodología cualitativa (Teoría Fundamentada) con los socios promotores de CTA creadas en el periodo 2006-2007, todas ellas localizadas en la Comunidad Valenciana.

Palabras clave: Proceso de creación de empresas, Cooperativas de Trabajo Asociado, Teoría Fundamentada, Redes, Viabilidad.

\footnotetext{
1 Florida Universitaria (Centro Adscrito a la Universidad de Valencia). Dirección de correo electrónico: rjcunyat@florida-uni.es.

REVESCO N 119 - Tercer Cuatrimestre 2015 - ISSN: 1885-8031 - www.ucm.es/info/revesco

http://dx.doi.org/10.5209/rev_REVE.2015.n119.49067

Fecha de recepción: 27/05/2014

Fecha de aceptación: 12/03/2015
} 
Claves Econlit: D710, M100, M130, O120.

\title{
NETWORKS AS A KEY FACTOR FOR THE CONSOLIDATION OF NEW WORKER COOPERATIVES
}

\begin{abstract}
In the last years of crisis and job losses, interest in boosting the dynamism and growth of the economy in a geographic area through the creation of new firms has been increasing. Worker cooperatives (CTA) represent a business model in which the economic objectives are combined with social, achieving growth based on employment, social equality and equity. New CTA need knowledge to its consolidation, one part of which comes from the training and experience of its promoters, and another of the interpersonal and interorganizational relationship within its economic and social environment. The survival and growth of the CTA will be very influenced by their ability to maintain and extend their networks. This paper aim to contribute to the knowledge of the different types of networks that is related to a cooperative from birth to consolidation, as well as the impact on their survival and growth. We have made a total of 36 in-depth interviews using a qualitative methodology (Grounded Theory) with promoters of CTA created in the period 2006-2007, all of them located in the Valencian Community.
\end{abstract}

Keywords: Entrepreneurship, Worker cooperatives, Grounded theory, Network, Viability.

\section{INTRODUCCIÓN}

En estos últimos años de crisis y destrucción masiva de empleo, el interés por impulsar la economía a través de la creación de nuevas empresas ha ido en aumento, convirtiéndose en muchas ocasiones en objetivo principal de las administraciones públicas. Este interés, condicionado en ocasiones por la repercusión en la generación de nuevos puestos de trabajo, y en otras por la contribución al desarrollo de nuevas ideas productivas que benefician a los consumidores, se ha volcado en la aparición de medidas de apoyo para la generación y puesta en marcha de nuevos proyectos empresariales.

No obstante, la tasa de mortalidad en los primeros años de vida de una empresa suele ser muy elevada, de forma que el efecto positivo que se prevé viene determinado por sus supervivencia; y ésta viene determinada tanto por las propias características de la empresa 
como del elemento humano, es decir, de los rasgos propios del empresario y de su cualificación.

Actualmente las CTA pueden desempeñar un papel clave en la recuperación económica de un país, puesto que se trata de empresas que contribuyen al desarrollo territorial, que no se deslocalizan, permitiendo una mayor integración territorial y cohesión social, generando empleo estable y de calidad en el lugar dónde se ubican (Lejarriaga, Bel, Martin, 2013). Se trata de empresas que favorecen el desarrollo local al estar particularmente ligadas al territorio, ya que éstas están vinculadas directamente a las personas que la integran, y éstas lo están al territorio (Garcia-Gutiérrez, 1991). De esta forma podemos justificar la cooperativa como una fórmula idónea para el desarrollo potencial endógeno de una zona (Monzón y Morales, 1998).

Sin embargo, los primeros años de vida de una cooperativa configuran sus posibilidades futuras, puesto que pueden nacer con importantes defectos relacionados con el débil conocimiento del mercado y del propio funcionamiento de éste (Cuñat y Coll, 2006). Todos estos aspectos hacen que el estudio del conjunto de relaciones que los cooperativistas mantienen con otros agentes sea un tema de interés, pues las redes permiten desarrollar un capital social que proporciona acceso a recursos, provee de información relevante, y es una fuente de competitividad y legitimidad; facilitando la innovación y el reconocimiento de oportunidades.

Las CTA son aquél tipo de empresas que agrupan como socios ordinarios a personas físicas que, mediante su trabajo en común, realizan cualquier actividad económica de producción de bienes y servicios para terceros, y tienen como objetivo, entre otros, crear y mantener puestos de trabajo sustentables, generando riqueza, para mejorar la calidad de vida de los socios, dignificar el trabajo humano, permitir la autogestión democrática de los trabajadores y promover el desarrollo comunitario y local.

La decisión de crear una CTA se corresponde con una iniciativa emprendedora desarrollada por un grupo de personas, que tienen como objetivo establecer relaciones laborales a largo plazo dentro de supuestos de democracia interna y solidaridad entre sus miembros. Esto les diferencia de un proyecto empresarial realizado bajo un modelo capitalista tradicional (Orellana, Martínez de Lejarza, 2013). Se trata de empresas que contribuyen al desarrollo territorial, y que actúan de forma socialmente responsable. Las CTA suelen 
permanecer enraizadas en su lugar de origen, lo que permite una mayor integración y cohesión social, y desempeñan un papel relevante por lo que respecta al desarrollo de mercados competitivos (Martín, S., Lejarriaga, G., 2011).

En estas empresas la identidad entre empresario y empresa se entremezcla, pues tienen un carácter personalista; existiendo por tanto poca distinción entre las redes de los individuos y las redes de empresa, especialmente en sus primeros años de vida. Los emprendedores cooperativistas presentan un alto nivel de pasión y compromiso con sus ideas, desarrollando una alta adaptabilidad a los cambios del entorno; esto les faculta para involucrar en sus proyectos a una gran variedad de grupos de interés; sin embargo es fundamental en la puesta en marcha de nuevos emprendimientos disponer de una sólida red de apoyo para canalizar sus iniciativas e impulsar sus actuaciones (Morales, Ariza, Muñiz; 2012).

El objetivo del presente trabajo se centra en identificar el tipo de redes sociales que utilizan las cooperativas a lo largo de sus primeros años de vida, y en concreto desde su nacimiento hasta la consolidación; así como el impacto que tienen éstas en la supervivencia y crecimiento de la cooperativa.

Se desarrolla un enfoque en el que se analizan las redes como variables dependientes, y cuyo objeto de análisis se centra en identificar cómo la evolución del proceso de creación de una empresa afecta al uso de uno u otro tipo de redes, y cómo éstas contribuyen al rendimiento de la cooperativa. Este enfoque sigue la misma línea que la formulada por Lechner y Dowling (2003) con referencia al estudio del tipo de relaciones que se desarrollan dentro de las redes que trazan los emprendedores, a lo largo de la secuencia que sigue el proceso de creación de la empresa.

Los resultados obtenidos en la investigación permitirán a las cooperativas de trabajo asociado orientar sus esfuerzos hacia la construcción de redes que favorezcan su consolidación en el mercado.

Para realizar el estudio se recogerán datos de cooperativas de trabajo asociado creadas en la Comunidad Valenciana en los años 2006-07, pues son aquellas cooperativas que llevan actualmente 7 años de funcionamiento, que es el periodo dónde se puede considerar, según la literatura, como empresa de reciente creación (Timmons, 1990; Vesper, 1990); tomando como base de datos las CTA presentes en el registro de Cooperativas de la Comunidad Valenciana en el año 2013, depurado y actualizado. 
El análisis del contraste empírico se realizará aplicando una metodología cualitativa, como la Teoría Fundamentada, que nos permite estudiar no solo el comportamiento de la cooperativa, sino también conocer todos aquellos aspectos que influyen en la conducta de los individuos que gestionan la empresa (Douglas, 2004).

El trabajo se estructura como sigue. En el apartado segundo se identifican y describen las fases del proceso de creación de una cooperativa de trabajo asociado, así como su relación con las redes interpersonales e interorganizacionales formadas durante el proceso. El punto 3 identifica los componentes de cada una de las redes que influyen en el proceso emprendedor, al tiempo que describe la metodología empleada en el análisis y el proceso de trabajo realizado para alcanzar los objetivos del estudio. En el apartado 4 se muestran los resultados y aspectos más relevantes obtenidos en el presente trabajo; y finalmente en el último de los apartados se especifican las principales conclusiones del análisis realizado.

\section{EL PROCESO DE CREACIÓN DE EMPRESAS Y LAS REDES}

El proceso de creación de una empresa puede ser estudiado desde las diferentes etapas que lo configuran. Muchos autores coinciden en iniciar el proceso estudiando aquellos factores que predisponen a una persona a crear su propio negocio y finalizarlo cuando la empresa se considera consolidada en el mercado. En este sentido nos vamos a apoyar en el modelo de creación de cooperativas de trabajo asociado desarrollado por Cuñat (2005), y que como podemos ver en la figura 1 se compone de cuatro fases:

- FASE I: Acontecimientos Desencadenantes. En esta fase el empresario cooperativista se predispone a crear su propia empresa, y en ella se analizan los factores que desencadenan el proceso de creación.

- FASE II: Concepto de Negocio. Dónde se describe la secuencia que lleva al inicio de la creación física de la cooperativa.

- FASE III: Formalización de la Empresa. Dónde se crea físicamente la empresa a través de un proceso de obtención de los recursos necesarios.

- FASE IV: Consolidación del Negocio. Es el tiempo que transcurre entre la creación física de la cooperativa y su consolidación en el mercado. En nuestro caso hemos estimado un periodo de 6-7 años.

Durante el desarrollo de las diferentes fases antes nombradas, los emprendedores van haciendo uso de diferentes contactos en los que se apoyan para desarrollar su proyecto, en 
este sentido crean diferentes tipos de redes. Para Johannisson y Monsted (1997) una red se puede definir como un conjunto de relaciones directas entre el empresario y distintas personas de su entorno socio-económico y familiar, y que está asociada con la persona que mantiene relaciones, siendo una formación social que existe sólo si la persona la conoce y la usa. "Las redes son uno de los capitales más importantes que alguien puede poseer, pues proporcionan poder, información, conocimiento y capital, así como otras redes” (Elfring y Hulsink, 2003, p. 409).

Figura 1. Proceso de creación de empresas cooperativas de trabajo asociado

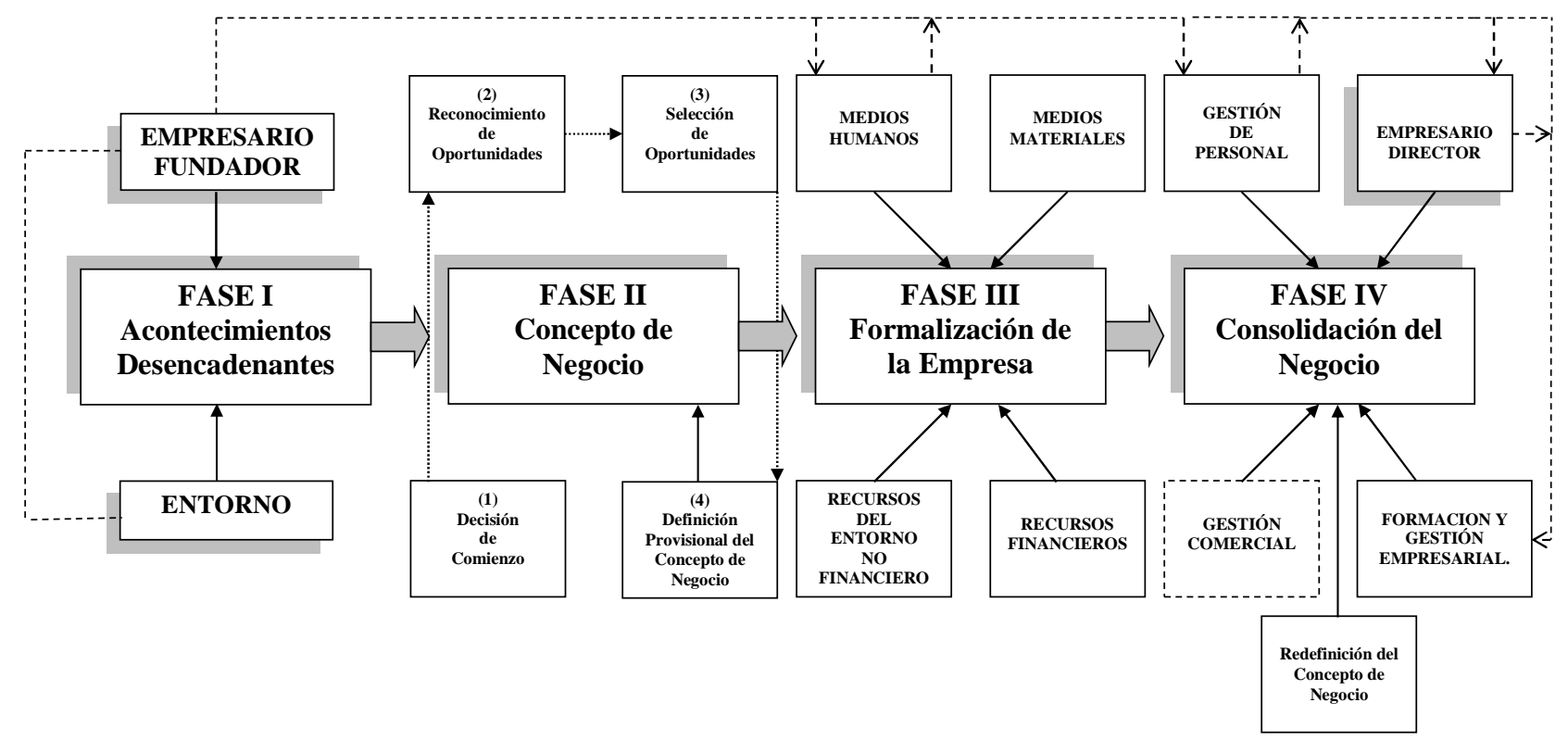

Fuente: Cuñat (2005)

Según Venkataraman y Van de Ven (1998), la supervivencia y crecimiento de una empresa nueva depende de su habilidad por mantener y extender sus redes. Por otra parte, la sinergia que se produce entre las redes y las empresas puede generar capacidades de aprendizaje, que repercute en su habilidad para innovar, introducir nuevos productos y servicios, así como descubrir nuevas oportunidades (Elfing y Hulsink, 2003). Incluso "las redes actúan cómo un amortiguador contra posibles sorpresas en el mercado globalizado" (Madsen, 2007, p. 191).

Los emprendedores que generan nuevas cooperativas necesitan conocimiento, una parte del cual proviene de su formación y experiencia; sin embargo, esto no es suficiente para garantizar la sostenibilidad y crecimiento de la cooperativa, por lo que necesita de conocimiento que adquiere de su entorno social y económico. De esta manera, la cooperativa 
puede movilizar conocimiento y recursos de otros para obtener beneficios a través de redes que se convierten en un verdadero capital social. Cuantas mejores conexiones tienen, mayores beneficios pueden obtener (Knoke y Burt,1986).

Estas redes, de naturaleza interpersonal por una parte (formadas por lazos de tipo familiar, amigos, colegas, contactos de negocios y afiliaciones a asociaciones profesionales), y por otra de carácter interorganizacional (relacionadas con el mercado) constituyen lo que algunos autores califican como mix relacional (Johannisson, 1998). Para una mejor comprensión de los tipos de redes que vamos a analizar, vamos a hacer referencia a la clasificación presentada por Schott y Cheraghi (2012), y que se describe en la figura 2.

Para Casson y Della (2007) el crecimiento de un emprendimiento se logra a partir del fortalecimiento de las redes interorganizacionales. Esto es especialmente importante en gran parte de las CTA, pues en estas redes intervienen una gran diversidad de interlocutores (otras cooperativas, administraciones locales, empresas sin ánimo de lucro, etc.) con las que va a ser necesario interactuar y coordinarse (Zandonal, Pezzini, 2004).

No obstante, la parte de relaciones interpersonales siempre es fundamental, debido a que las redes interorganizacionales se mantienen en la práctica a través de las comunicaciones interpersonales entre los representantes de las organizaciones; un cambio en las personas puede influir en las relaciones entre las empresas, si los sucesores no son capaces de manejar de forma apropiada estas relaciones; y esto a pesar de que las cooperativas son redes de empresarios (en nuestro caso también trabajadores) que buscan unidos las ventajas de la unión y la obtención de sinergias en cualquier actividad que realizan (Bel y Ausín, 2007).

\section{METODOLOGÍA Y PROCEDIMIENTO DE TRABAJO}

El presente trabajo supone una contribución a la investigación científica en el campo de la creación de empresas y la teoría de redes, por cuanto se recurre a un método inductivodeductivo como es la Teoría Fundamentada para profundizar en su estudio, de aplicación novedosa en esta disciplina, pero no en otras del área de las ciencias sociales. Por citar algunos ejemplos podemos encontrar aplicaciones sobre la gestión turística y hotelera (Connell y Lowe, 1997); Management (Henwood y Pidgeon, 1995); Creación de Empresas (Douglas, 2004); investigación en Dirección de Empresas (Locke, 2001); Innovación (Lowe, 1995); fusión de empresas (Lowe, 1998); Gestión Directiva (Partington, 2000); internacionalización (Etemad, 2004); Recursos Humanos (Clinton, Totterdell, Wood; 2006); 
entre otros.

La Teoría Fundamentada ofrece la posibilidad de analizar el fenómeno de la Creación de Empresas con un nivel de detalle adecuado que facilita el conocimiento de todos aquellos aspectos relevantes sobre la utilización de las redes. De esta forma podemos conocer las relaciones clave en el proceso de creación de una empresa pertenecientes tanto al empresario como a su entorno. La investigación a través de la Teoría Fundamentada es una metodología apropiada para el análisis tanto de los aspectos económicos del proceso como de las conductas de sus actores.

Figura 2. Redes del emprendedor

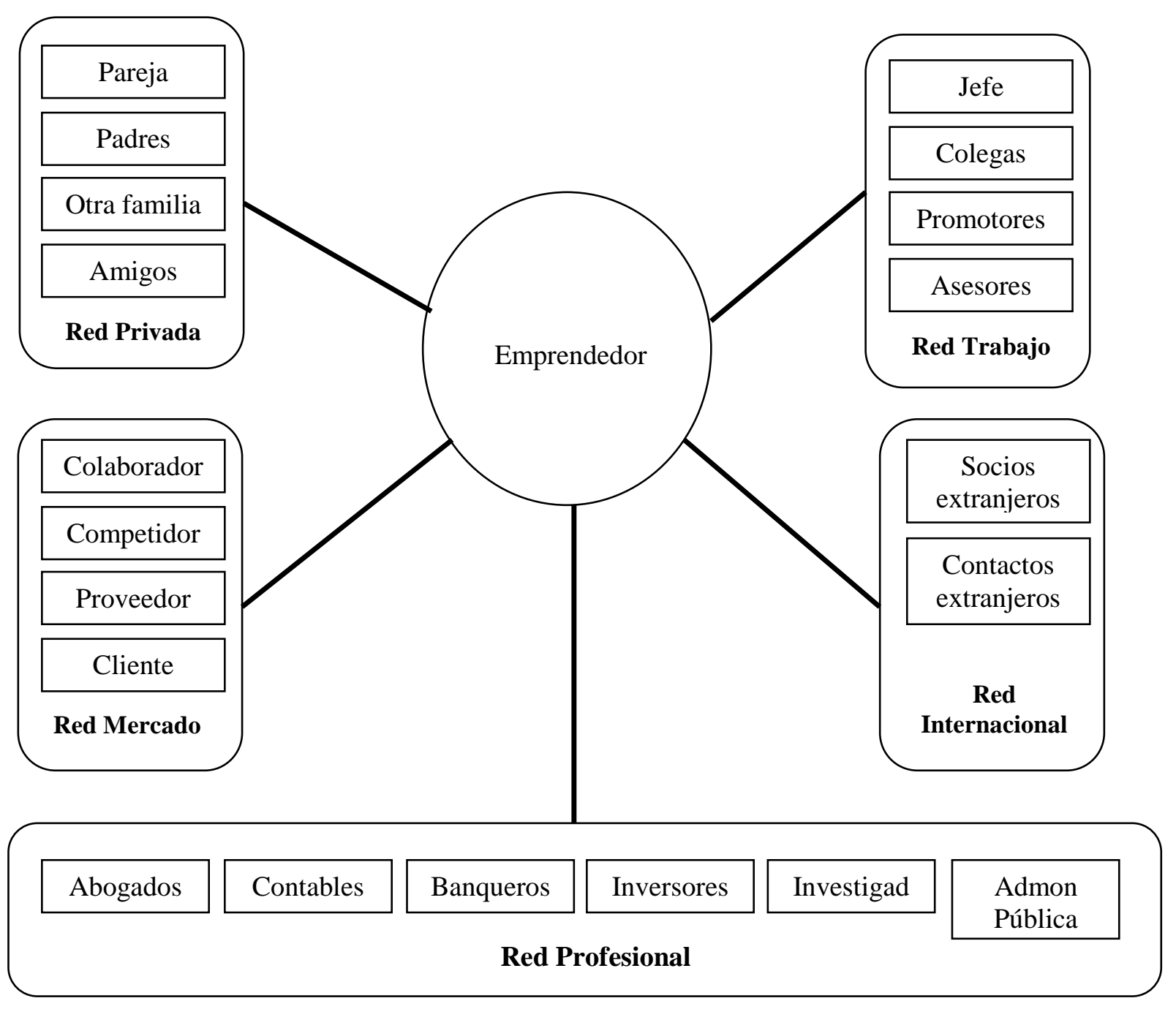

Fuente: Schott y Cheraghi (2012, pág. 221)

La Teoría Fundamentada nos permite construir teorías, conceptos, hipótesis y proposiciones partiendo directamente de los datos y no de supuestos a priori, de otras investigaciones o de marcos teóricos existentes. La teoría generada se desarrolla 
inductivamente a partir de un conjunto de datos. Si se hace adecuadamente, esto significa que la teoría resultante cuadra al final con la realidad objeto de estudio (Glaser y Strauss, 1967). Ello contrasta con una teoría derivada deductivamente de una gran teoría, sin la ayuda de datos, y que podría por lo tanto no cuadrar con la realidad.

La Teoría Fundamentada es una técnica de investigación cualitativa en el que la teoría emerge desde los datos (Glaser y Strauss, 1967). Es una metodología inductiva que tiene por objeto la identificación de los procesos sociales básicos (PSBs) como punto central de la teoría. A través de esta metodología podemos descubrir aquellos aspectos que son relevantes de una determinada área de estudio (Strauss y Corbin, 1990).

La Teoría Fundamentada parte de la orientación de caso de tipo comparativo. Casos similares con muchas variables pero con diferentes respuestas son comparados para ver dónde puede encontrarse la clave de las diferencias. Este procedimiento se basa en el método de diferencias de John Stuart Mills.

Igualmente, los casos que obtienen la misma respuesta son examinados para ver qué condiciones tienen todos en común. Las estrategias principales para desarrollar la Teoría Fundamentada son dos: a) el método comparativo constante y b) el muestreo teórico.

A través del método comparativo constante el investigador simultáneamente codifica y analiza datos para desarrollar conceptos. Mediante la comparación continua de incidentes específicos de los datos, el investigador refina esos conceptos, identifica sus propiedades, explora sus interrelaciones y los integra en una teoría coherente.

Desde el muestreo teórico, el investigador selecciona nuevos casos a estudiar según su potencial para ayudar a refinar o expandir los conceptos y teorías ya desarrollados. La recolección de datos y el análisis se realizan al mismo tiempo.

El objetivo de la Teoría Fundamentada es, por tanto, el descubrimiento de una teoría explicativa comprensiva acerca de un fenómeno particular. Las técnicas y procedimientos analíticos permiten al investigador desarrollar una teoría substantiva que es significativa, compatible con el fenómeno observado, generalizable, reproducible y rigurosa. La Teoría Fundamentada es deductiva e inductiva. Inductivamente, la teoría surge desde las observaciones y genera datos. Esta teoría puede ser entonces examinada empíricamente para desarrollar pronósticos o predicciones desde unos principios generales. 
La generación de teoría a partir de la inducción favorece la explicación exacta del fenómeno estudiado, más que su generalización. El poder explicativo de la Teoría Fundamentada está en desarrollar la habilidad de poder explicar un suceso; por ejemplo qué podría ocurrir en un negocio, o a un empresario, a partir de incidentes procedentes del campo de estudio, y ayudar a comprender aspectos propios de la estructura analizada (Gibson, Gregory y Robinson; 2005).

En la figura 3, podemos ver los componentes básicos de la Teoría Fundamentada y las interrelaciones entre sus variables siguiendo las indicaciones iniciales de Glaser y Strauss (1967), y los posteriores trabajos desarrollados por Glaser (1992, 2002, 2004), Strauss (1987), y Strauss y Corbin (1990).

En este modelo podemos identificar dos niveles de explicación. Por una parte la perspectiva horizontal muestra los elementos básicos del modelo. En este nivel se pretende mostrar los principales ingredientes que influyen en la transformación de los datos; es decir: tipos de códigos conceptuales, formas de codificación y tipos de teoría emergente.

El segundo nivel describe el modelo a partir de los elementos claves asociados a acciones o tareas de la Teoría Fundamentada, y que representan en sí mismos situaciones que vinculan a los distintos elementos en la generación de teoría. De esta forma, el muestreo teórico, el método de análisis comparativo constante, la saturación teórica, la clasificación teórica, el establecimiento de los procesos sociales básicos y la escritura teórica, componen los elementos dinámicos del modelo.

La significatividad del modelo viene marcada por las conexiones entre cada uno de los elementos en un mismo nivel, y con el resto de elementos del segundo nivel donde los procesos de producción de datos y de análisis inductivo comparativo se suceden indistintamente, hasta los criterios de saturación conceptual que permiten identificar los PSBs.

Inicialmente el proceso comienza con la identificación de un área de interés a explorar. La misión del investigador es construir su propia teoría desde la base. Glaser (1978) comenta que los datos pueden provenir de distintas fuentes directas como la entrevista y el cuestionario, e indirectas como la experiencia percibida por el investigador.

Con la Teoría Fundamentada el tamaño de la muestra está relacionada directamente con la teoría. Es un parte más del proceso de recogida de datos y análisis. La recogida de 
datos va configurando el tamaño de la muestra final. Ésta viene determinada por el desarrollo de las categorías identificadas y la teoría emergente (Coyle, 1997). El investigador no conoce inicialmente el tamaño final de la muestra (Glaser, 1992; Coyle, 1997). La ampliación de la muestra se produce cuando se necesita más información para la investigación. A medida que los conceptos son identificados y la teoría comienza a desarrollarse se necesita incorporar más datos procedentes de nuevas empresas y situaciones para encontrar fundamentos más sólidos.

Figura 3. Componentes básicos de la Teoría Fundamentada

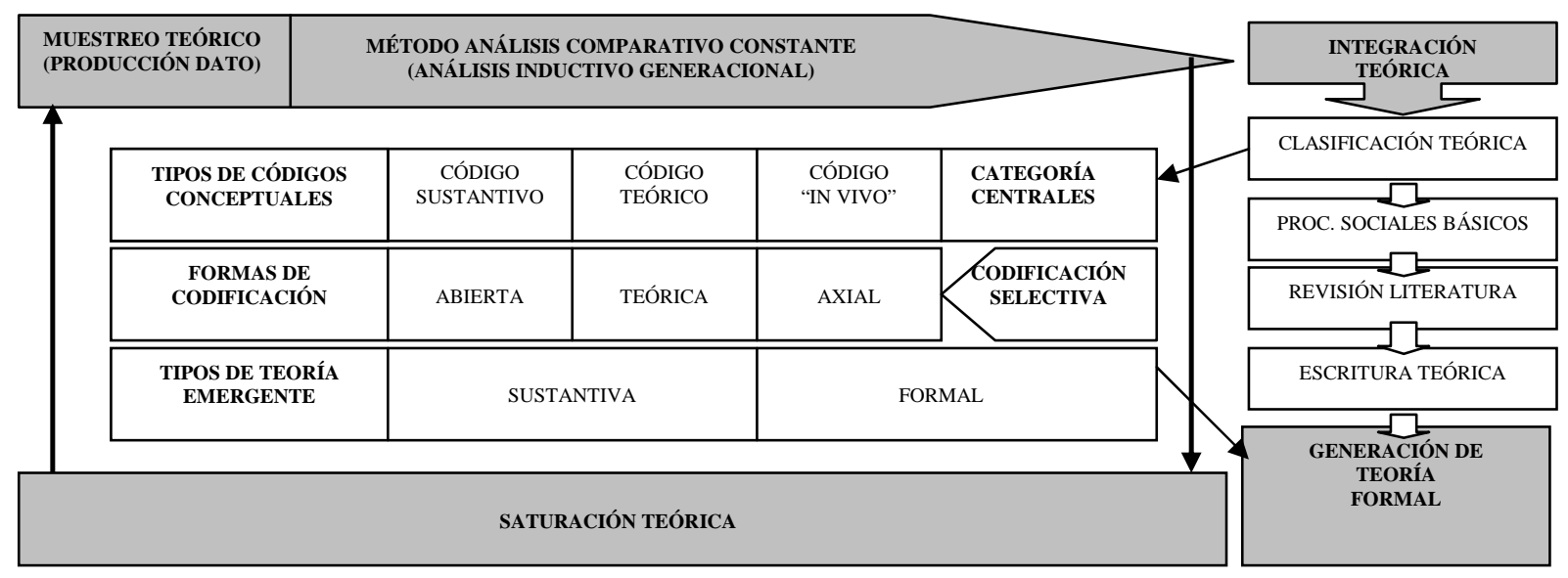

Fuente: Adaptado de Carrero (1998, p. 260)

Esto se conoce como muestreo teórico, que es el proceso de recogida de datos que generan teoría desde el mismo momento en que se recogen, se codifica y analiza la información proporcionada y se decide qué datos serán recogidos en las siguientes entrevistas y dónde se encontrarán, con relación a desarrollar la teoría tal como va emergiendo. Este proceso de recolección de datos es controlada por la teoría emergente (Glaser, 1978). La credibilidad final de la teoría generada depende menos del tamaño de la muestra que de la riqueza de información recogida y las habilidades analíticas del investigador.

En la Teoría Fundamentada se produce simultáneamente la recogida y análisis de los datos. Desde las primeras 3 o 4 entrevistas se van generando códigos, identificando qué información se desea ampliar o incidir. Las sucesivas entrevistas van definiendo los aspectos sobre los que se volcará la búsqueda de información adicional (Glaser y Strauss, 1967).

El proceso comienza con códigos abiertos sobre los datos recogidos, que dirigen la muestra en todas direcciones hasta el descubrimiento de variables centrales fuertemente respaldadas por los datos. Cuando este nivel se consigue la muestra llega a ser selectiva y se 
dirige hacia los temas que son centrales en la teoría emergente. De acuerdo con Glaser (1978), el investigador debe estar preparado para mantenerse abierto a la posibilidad de nuevas direcciones en la recogida de la información; por ejemplo, modificar el estilo de la entrevista, los informantes, etc. Sólo cuando ya no existen posibles conceptos emergiendo desde los datos, deberá el investigador dejar de buscar más información. En otras palabras, cuando los datos están saturados. En este sentido, el procedimiento general para definir la muestra final consiste en identificar códigos de los datos obtenidos desde el comienzo en la recogida de información, y lograr su saturación a través del análisis comparativo constante (Glaser, 1992).

La comparación explora las diferencias y similitudes a lo largo de los incidentes identificados dentro de la información obtenida. El análisis explícitamente compara cada incidente proporcionado por los datos con otros incidentes parecidos que pertenecen a la misma categoría, explorando sus similitudes y diferencias (Spiegelberg, 1994). El investigador debe asegurarse que la comparación constante va caracterizando el proceso.

La saturación teórica se logra recogiendo información hasta que ya no surgen nuevas evidencias o sucesos que pueden informar del desarrollo del proceso. No hay una norma clara de corte, pero es importante saturar la información obtenida. Cuando se van produciendo situaciones similares a las ya registradas, el investigador deberá confiar en que la categoría se ha saturado (Glaser y Strauss, 1967).

Todos los conceptos se agrupan en categorías descriptivas. Son entonces re-evaluados por sus interrelaciones, y a través de una serie de pasos analíticos son gradualmente incluidos en más altas categorías o categorías centrales, que sugieren una teoría emergente. La teoría que emerge del análisis de las categorías nos permite hacernos una idea de la situación (Jezewski, 1995). De acuerdo con Glaser (1978), una categoría central representa un patrón de conducta. Es la sustancia de lo que reflejan los datos que se han recogido. Una categoría central debería saturarse tan pronto como fuera posible para ver su poder de explicación, apoyándose en una completa muestra teórica que maximice las diferencias entre los datos.

La meta de la Teoría Fundamentada es generar teoría que describa para un patrón de conducta qué es relevante y problemático de las situaciones que son estudiadas. La teoría identificada se puede agrupar en PSBs. Éstos agrupan situaciones similares que se producen en diferentes momentos del tiempo. 
Cuando la teoría está completamente desarrollada, el investigador se enfrenta con el desafío de escribirla. Inicialmente comenzará con una clasificación teórica y continuará con la escritura. Esta fase está fuertemente cargada de dilemas sobre la estructura que deberá tener el relato, el grado de detalle metodológico que debe incluir, y la cantidad de datos a presentar para que garanticen la consistencia de la teoría en base a la información obtenida.

Por sus características, la Teoría Fundamentada requiere iniciar la generación de la teoría sin hipótesis, ni ideas preconcebidas sobre el área substantiva objeto de estudio. Se recomienda, por tanto, iniciar el estudio sin una revisión previa de la literatura, con el fin de garantizar que las hipótesis están siendo generadas desde los datos y, por tanto, conformadas en su nivel conceptual como teoría fundamentada. La revisión de la literatura se realiza entre la fase de clasificación teórica y la escritura teórica.

La integración de la literatura se produce en el texto del trabajo y en las notas a pie de página. A través de las citas se extiende la teoría propia hacia distintas teorías elaboradas por otros autores. Uno de los objetivos más importantes en la revisión de los trabajos es la integración de la teoría generada en la literatura existente.

Por lo que respecta al procedimiento de trabajo se partió de la población total de Cooperativas de Trabajo Asociado creadas durante los años 2006 y 2007 en la Comunidad Valenciana, tal como muestra la tabla 1, y que seguían activas. El apoyo de la Federación Valenciana de Cooperativas de Trabajo Asociado (FEVECTA) y el asesoramiento de expertos del mundo cooperativista nos permitió analizar la población, con objeto de seleccionar una muestra que pudiera abarcar una diversidad de áreas de actividad y colectivos, y fuera representativa.

Se seleccionaron 36 empresas cooperativas de trabajo asociado de un total de 214 creadas durante los años 2006 y 2007 pertenecientes a diferentes sectores empresariales, presentes en la base de datos del Registro de Cooperativas de la Comunidad Valenciana, y activas en el año 2013. 
Tabla 1. Población total de CTA creadas durante los años 2006-07 activas en la Comunidad Valenciana

\begin{tabular}{|c|c|c|c|c|}
\hline & Alicante & Castellón & Valencia & Total \\
\hline Número de CTA & 76 & 10 & 128 & 214 \\
\hline
\end{tabular}

Fuente: Registro de Cooperativas de la Comunidad Valenciana (2013). Depurado y Actualizado

La figura 4 muestra un periodo de investigación que abarca desde Enero a julio del 2013. Durante este periodo, se combinan fases de recogida de datos y fases de análisis. Hay que tener presente que los fundamentos del muestreo teórico permiten delimitar, a partir del análisis inicial de los primeros datos, qué unidades de análisis serán el foco de atención y qué informantes son los más adecuados.

En Febrero del 2013 comenzó el desarrollo de entrevistas al conjunto de equipos promotores de las cooperativas. En esta primera fase (ver figura 4), realizamos diez entrevistas a empresas diferentes, seleccionadas de forma aleatoria y pertenecientes al grupo de cooperativas de la muestra propuesta. La información de las entrevistas fue grabada y transcrita por los analistas que conformaban el grupo de investigación.

Después de la recogida y grabación de la información, se procedió a su análisis utilizando el programa informático MAXQDA, que nos permitía almacenar todas las trascripciones de las entrevistas, memos, y las variables descriptivas más importantes de cada una de las cooperativas. Este programa facilitó un importante ahorro de tiempo en el manejo de los datos, afectando a la fiabilidad de los diseños resultantes de la investigación, puesto que deja un rastro que puede posibilitar su auditoría (falseabilidad) al tiempo que dota de credibilidad los hallazgos (Fielding y Lee, 1998). Finalmente permite al analista enfrentarse directamente al texto, sin una plantilla de códigos (libro de claves) o alguna estructura elaborada a priori. Así mismo ayuda al investigador a no excluir, si así lo desea, de la exploración ninguna información.

No obstante, hay que considerar como limitaciones a la utilización de estos tipos de programas que la capacidad analítica depende de la formación teórica y metodológica del investigador. Por ello, el uso óptimo de esta herramienta se alcanzará en mayor o menor grado en función de dicha capacidad (Valles, 2005). 
Figura 4. Diseño del estudio empírico. Procedimiento de recogida y análisis de datos

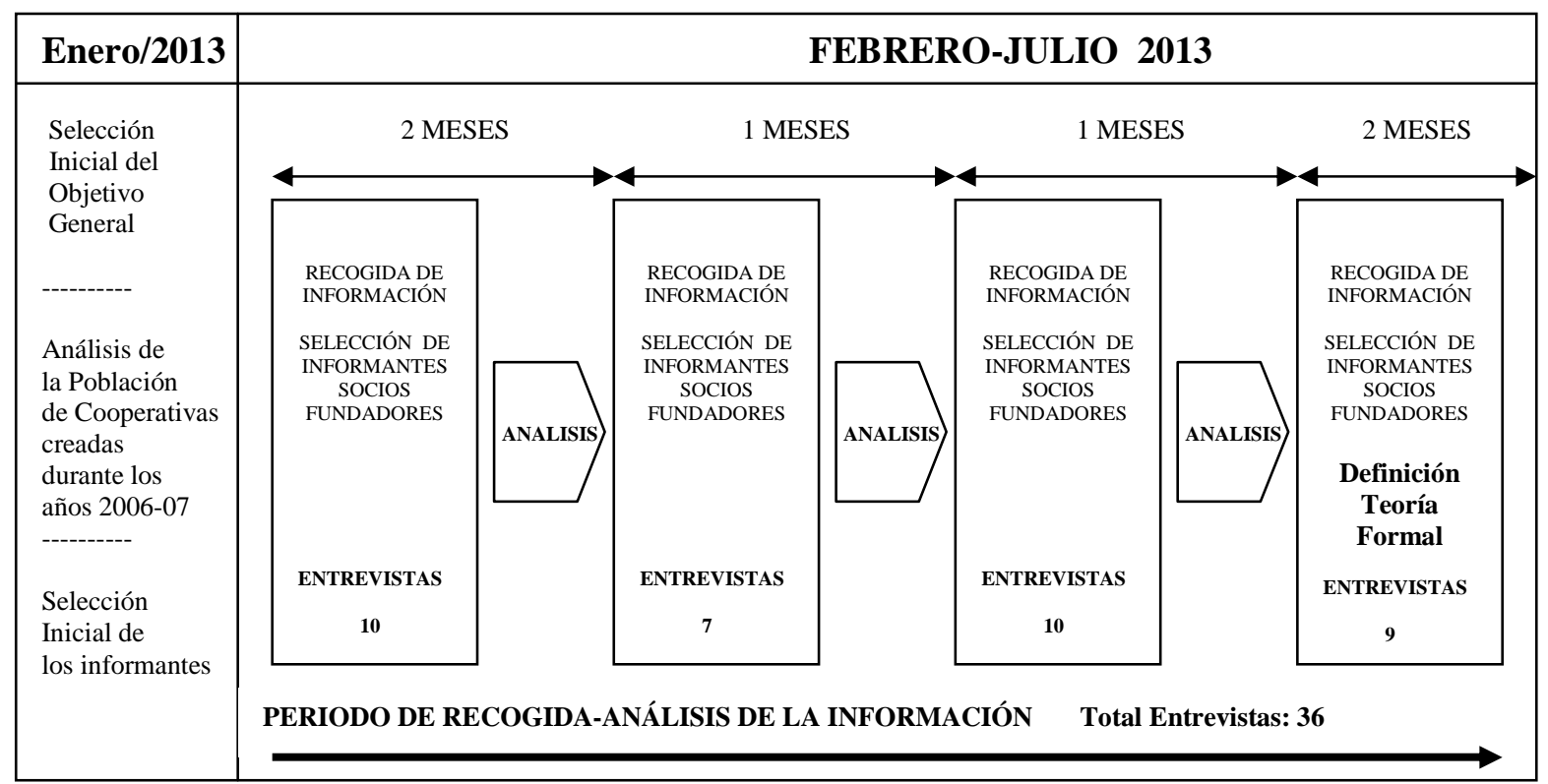

Fuente: Elaboración Propia

Siguiendo con el proceso, la selección de nuevos informadores, así como el foco de atención de las siguientes entrevistas, estaban marcados por los resultados obtenidos en cada uno de los análisis que se iban realizando. A medida que se producían nuevas entrevistas y analizando la información, muchas de los datos identificados se saturaban.

Se incrementó en cuatro el número de entrevistas que inicialmente fueron seleccionadas, y a finales de julio del 2013 el grupo de analistas estaba seguro de la saturación de los datos obtenidos, pues ya no aparecían nuevas informaciones. La información en las diversas sesiones de discusión se repetía y no existían datos que pareciesen informar de nuevas categorías o propiedades, y los procesos sociales básicos parecían quedar identificados.

De esta forma, se dio por concluido el muestreo teórico; con un total de 36 empresas cooperativas entrevistadas, cuyos principales datos quedan reflejados en la tabla 2 a nivel informativo. En ella podemos ver las características de la muestra final; con un porcentaje mayor de cooperativas del sector servicios, manteniendo así la proporción de sectores presente en la población total de cooperativas registradas en la Comunidad Valenciana en el periodo seleccionado.

El procedimiento descrito nos permite observar como el proceso de análisis no se puede considerar como la fase final de la investigación. Al contrario, el análisis y codificación de los incidentes encontrados en los datos se intercala, a modo de eslabón, entre la recogida 
de datos y su discusión e interpretación por los analistas; en un continuo ir y venir de los datos hacia las categorías generadas y viceversa.

Tabla 2. Características de la muestra final

\begin{tabular}{|l|c|c|c|c|c|c|}
\hline Sector de Actividad & $\begin{array}{c}\text { Número } \\
\text { Empresas }\end{array}$ & \multicolumn{2}{|c|}{$\begin{array}{c}\text { Número } \\
\text { Socios } \\
2006-07\end{array}$} & \multicolumn{2}{|c|}{$\begin{array}{c}\text { Número } \\
\text { Socios } \\
2013\end{array}$} & $\begin{array}{c}\text { Incremento } \\
\text { Medio de la } \\
\text { Plantilla Total }\end{array}$ \\
\hline & & Total & $\begin{array}{c}\text { Media } \\
\%\end{array}$ & Total & $\begin{array}{c}\text { Media } \\
\%\end{array}$ & $\%$ \\
\hline Agricultura & 2 & 6 & 3 & 5 & 2,5 & $-16,6$ \\
\hline Industria y Construcción & 9 & 53 & 5,9 & 66 & 7,3 & 23,7 \\
\hline Comercio & 2 & 6 & 3 & 9 & 4,5 & 50 \\
\hline Servicios & 23 & 104 & 4,5 & 182 & 7,9 & 75,5 \\
\hline TOTAL & 36 & 169 & 4,7 & 262 & 7,3 & 55,3 \\
\hline
\end{tabular}

Fuente: Elaboración Propia

Esta forma de proceder, siguiendo un proceso repetitivo e interactivo, garantiza la generación de teoría fundamentada desde los datos, y además permite identificar, a partir de la saturación de las categorías, el momento en el que la muestra ha sido recogida en su totalidad y si es adecuado cesar la recogida de los datos en el campo de estudio.

\section{RESULTADOS Y DISCUSIÓN}

Llegados a este punto, comenzamos el proceso de integración teórica de toda la información obtenida. Como paso previo a la generación de la teoría formal, y siguiendo uno de los procedimientos propuestos por Glaser (1978) procedimos a dar cuenta de las realidades encontradas, las cuales se alimentaban directamente de los datos procedentes de la investigación de una manera dinámica y abierta. La teoría substantiva generada nos ayudó a reflexionar sobre las conclusiones a las que íbamos llegando a lo largo de todo el proceso de recogida y análisis de la información, y sirvió de nexo estratégico en la formulación y generación de la teoría formal fundamentada.

Para un mejor entendimiento en el desarrollo de la teoría formal respecto al uso de las diversas redes en el proceso de creación de la cooperativa de trabajo asociado, hemos dividido los resultados obtenidos en función de cada una de las fases identificadas en el modelo descrito en la figura 1. 


\subsection{FASE I. Acontecimientos Desencadenantes}

Según Honing y Davidsson (2003) en la etapa previa al emprendimiento, la presencia de contactos fuertes relacionados con la familia y el entorno laboral (redes sociales) tiene una repercusión directa en el descubrimiento de oportunidades de negocio, al tiempo que proporciona legitimidad, confianza y consejo para seguir adelante en el proceso emprendedor; pues la familia y los amigos reducen la incertidumbre y aumentan la confianza en las posibilidades del proyecto de negocio (Aldrich, 1999).

En las cooperativas analizadas, la red privada supone en todos los casos un factor de apoyo en la decisión de crear la nueva empresa. En este sentido tiene un mayor peso el aspecto moral que económico. El último paso que da el emprendedor hacia la creación del negocio siempre viene reforzado por el soporte familiar. No siendo relevante el hecho de que los familiares fueran o no empresarios. La mayor parte de los entrevistados reconocen que no hubieran comenzado su negocio sin el apoyo de su red privada, y que ésta ha sido también importante en la continuidad de la empresa.

Por otra parte la red de trabajo también ha sido determinante para desencadenar el proceso emprendedor, especialmente porque la mayor parte de las cooperativas de trabajo asociado se generan a partir de la pérdida del empleo, y dónde se agrupan personas que mantienen contactos con su anterior empresa y colegas de trabajo; cuyas relaciones les proporcionan confianza y apoyo.

En esta primera fase no fueron nombradas ninguno de los otros tipos de redes que forman parte del modelo de redes de emprendimiento de referencia en este artículo.

\subsection{FASE II. Concepto de Negocio}

El proyecto de empresa comienza cuando el empresario reconoce una oportunidad en el entorno. Este reconocimiento puede venir a través de estímulos internos o externos procedentes de necesidades detectadas en el mercado. En esta etapa los promotores exploran primero las posibilidades de comenzar un nuevo negocio compartiendo la información con un reducido círculo de contactos (Greve y Salaff, 2003).

Jenssen y Koening (2002) indican que en esta etapa los emprendedores hacen uso de las redes sociales como vehículo para convencer a los proveedores de recursos de que su nueva idea es viable y legítima. Para conseguir esta legitimidad buscan reputación, pues a 
mayor reputación ganada más posibilidades de crear relaciones futuras; y contrariamente, la carencia de redes de reputación puede ser una barrera para el desarrollo de la nueva cooperativa. La conexión con profesionales y asociaciones empresariales incrementa la reputación de una empresa, lo que facilita el acceso a otros agentes, tales como proveedores y clientes, quienes se muestran más predispuestos a negociar al percibir que el riesgo en el intercambio futuro se va a reducir (Lechner, Dowling, Welpe; 2006).

En el estudio se muestra, durante esta segunda fase del proceso de creación de la cooperativa, el uso de redes como puntos importantes de apoyo, que permiten a los emprendedores mantener una mayor confianza en la viabilidad del proyecto, al tiempo que pueden compartir y contrastar su idea con el objetivo de ponerla en práctica. Sin embargo, hemos detectado que aquellos emprendedores que construyeron fuertes redes sociales de dependencia en esta etapa, en el futuro se han visto limitados en su capacidad para conseguir nuevos recursos por otros medios. Esto es debido a que estos fuertes lazos han impedido aumentar su red de contactos.

Es importante señalar que la mayoría de las cooperativas de la muestra analizada disminuían el poder de referencia de las redes sociales y canalizaban ya sus esfuerzos hacia las redes profesionales. Esta situación, tal como indica Salaff (2003) es propia de culturas dónde la familia no tiene una fuerte influencia sobre el individuo; lo que en España puede ser un aspecto de estudio por cuanto puede deberse a un cambio social producido en los últimos años.

En esta etapa los emprendedores se apoyan fuertemente en las redes profesionales, pues son los que les dan una mayor visibilidad., sobre todo si son poco conocidos en la zona. Se descubrió que aquellas cooperativas que habían desarrollado unas fuertes redes de reputación habían conseguido sus objetivos más pronto, y habían incrementado sus relaciones de mercado.

\subsection{FASE III. Formalización de la empresa}

Durante esta fase los emprendedores deben ser capaces de conseguir los recursos necesarios para el arranque y primeros pasos de la cooperativa. Esto les obliga a concentrar sus esfuerzos en aquellos contactos de los que pueden obtener recursos y compromiso (Greve y Slaff, 2003). Solymossy (2000) considera que las redes sólo serán útiles si la información 
que éstas proporcionan facilita el acceso a recursos críticos y valiosos capaces de proporcionar ventajas competitivas en el futuro.

El equipo de trabajo que se constituye en esta etapa se limita al grupo promotor. Dos son las circunstancias que condicionan este hecho: por una parte el interés de los fundadores en mantener la cohesión del grupo sin la entrada de personas que no participaron en la generación de la idea, y por otra el riesgo que supone contratar o hacer socio a otra persona en una situación donde la incertidumbre al futuro está presente. Por otra parte el tamaño inicial de las instalaciones es pequeño y se ajusta a la demanda que previsiblemente va a tener de inicio la empresa en función de las expectativas de los socios, y aunque el capital inicial de la cooperativa de nueva creación, en un porcentaje alto (entre el 47 al 73 por ciento), proviene de los recursos personales de los promotores, la búsqueda de recursos adicionales suele ser un aspecto difícil para la cooperativa (Cuñat, 2005).

En esta fase, y contrariamente a lo podríamos esperar, las cooperativas más exitosas procedentes de la muestra analizada se apoyan muy poco en las redes privadas y de trabajo, e intensifican su interés por los contactos procedentes de redes profesionales. Las cooperativas que construyeron fuertes lazos profesionales en sus comienzos incrementaron sus relaciones de mercado en el futuro; mientras que aquellas que no lo hicieron su supervivencia se vio afectada. Los lazos profesionales están formados por actores de alta visibilidad en el mercado, tales como profesionales o asociaciones empresariales, que facilitan a la cooperativa ser conocida en el mercado y alcanzar así un grado de reputación adecuado que permite establecer relaciones de confianza futura con proveedores y clientes.

Los resultados obtenidos en el estudio de esta fase estarían en la línea argumentada entre varios investigadores, entre ellos Schott y Cheraghi (2012), que indican que la menor importancia de las relaciones sociales en las culturas menos tradicionales induce a buscar relaciones fuera de ésta, incrementando el número de contactos y su diversificación, otorgando una mayor confianza en el mercado como apoyo para el lanzamiento del nuevo negocio.

\subsection{FASE IV. Consolidación del negocio}

En esta fase la cooperativa comienza su actividad, condicionando su consolidación al liderazgo por parte de algún socio promotor, la formación y capacitación del grupo promotor y las políticas de personal y comercial implementadas. El líder en este tipo de empresas juega 
un papel muy importante, puesto que debe canalizar los valores culturales de la cooperativa hacia un comportamiento estratégico adecuado, que armonice e integre la dimensión social y económica (Rodrigo, 1995).

En esta etapa será fundamental para la consolidación de la empresa seguir ganando legitimidad y reputación a través de relaciones de mercado y envío de señales al poseedor de recursos (Hoang y Antoncic, 2003), pues esto les permitirá reducir el riesgo que estos poseedores de recursos asocian con las ideas de la cooperativa y ganarse una imagen de confianza. Esta situación es especialmente importante en las cooperativas, pues la reputación de los emprendedores es importante en contextos de carácter local, donde son menos conocidos (Herrera, 2009).

En este periodo el éxito o fracaso de la cooperativa va a depender del grado en el que sean capaces de construir redes de mercado, al tiempo que hayan abandonado prácticamente las redes sociales y mantengan, pero de forma reducida, su red profesional. Las redes de mercado son redes de cooperación con competidores u otras empresas que permiten a la cooperativa obtener mejor información del mercado respecto a necesidades de desarrollar nuevos productos o bien conseguir mejor cuota de mercado o ganar clientes. Al mismo tiempo, pueden servir de transmisiones de experiencias o para el manejo de proyectos conjuntos con otras organizaciones.

Es importante destacar que los emprendedores cooperativistas más exitosos entienden, respecto a las relaciones con competidores, que los perjuicios de no colaborar superan los problemas que se podrían derivar del uso de información privilegiada de la empresa por parte de los competidores. Todos estos aspectos están en línea con los estudios realizados por Lechner y Dowling, 2003), que indican que la cooperación, especialmente con competidores regionales, incrementa la probabilidad de retorno de favores, y da acceso a recursos necesarios en los primeros años de vida de la empresa, cuando las ventas tienden a crecer de forma discontinua. El papel fundamental de las redes de colaboración se corresponde con el hecho de que favorecen las relaciones entre personas, contribuyendo a generar beneficios mutuos, confianza y orientación a la acción (Sanchis, 2001).

En el estudio se pudo observar que aquellas cooperativas que no mantenían redes de mercado o estas eran débiles no habían sido capaces de mejorar sus rendimientos, y las dificultades para mantenerse eran muy grandes. Es también importante resaltar que, durante 
esta fase de consolidación, el incremento de contactos de mercado aumenta el rendimiento de la cooperativa de forma significativa hasta tres contactos, pero a partir de esta cantidad los resultados no mejoran notablemente, e incluso a partir de un número más elevado de contactos (7-8 contactos) el efecto puede ser negativo. La razón que hemos identificado es la capacidad de los emprendedores cooperativistas para manejar el tamaño de la red, al tiempo que, al ser empresas locales en la mayor parte de ellas la diversidad disminuye la intensidad de los contactos, y por tanto los apoyos son más débiles.

En esta etapa sería de suponer que, moviéndonos en un entorno global, las cooperativas comienzan a construir redes internacionales que favorezcan su crecimiento y abran nuevas posibilidades para el desarrollo de nuevas oportunidades de mercado. Sin embargo la realidad nos ha mostrado que éstas no construyen este tipo de redes en esta etapa, lo que puede deberse a varios motivos: el carácter local de la mayor parte de ella, su tamaño o su aversión al riesgo. No obstante, los promotores cooperativistas indicaron que tenían intención de recurrir al mercado internacional una vez la empresa se consolidara en el mercado nacional.

\section{CONCLUSIONES}

La situación de crisis y destrucción masiva de empleo vivida en España en los últimos años ha provocado un alto interés por estimular la economía a través de la creación de nuevas empresas, no obstante la tasa de mortalidad en los primeros años de vida suele ser muy elevada, por lo que el efecto positivo de estos nuevos emprendimientos viene condicionado por su supervivencia; que a su vez está condicionada por las características de su componente humano y la interacción de la empresa con su entorno, marcada por las redes que permiten desarrollar un capital social que da acceso a recursos, información y oportunidades.

Las CTA son empresas que agrupan como socios ordinarios a personas físicas que, a través de su trabajo común, realizan cualquier actividad económica de producción de bienes y servicios para terceros. En estas empresas existe poca distinción entre las redes de los individuos y las redes de la empresa, pues tienen un carácter personalista. Los emprendedores cooperativistas presentan un alto nivel de pasión y compromiso con sus ideas, a la vez que son capaces de adaptarse rápido a los cambios del entorno.

Por otra parte, la aplicación de los principios cooperativos por las sociedades cooperativas les lleva a orientar su actividad buscando cubrir las necesidades e inquietudes de 
sus socios y demás grupos de interés, entre los que podemos contar la comunidad local, otras cooperativas, proveedores, clientes y sociedad en general (Puentes, Velasco; 2009).

La consolidación de una CTA dependerá de la forma en la que ésta ha gestionado su funcionamiento en cada una de las etapas del proceso de su creación, que en este artículo hemos considerado como cuatro: la fase de Acontecimientos desencadenantes, la fase de Concepto de Negocio, la fase de Formalización de la Empresa y la fase de Consolidación del negocio. En cada una de estas fases se realizan diferentes acciones, en las que parte de su éxito estará condicionado por el uso de los diferentes contactos y relaciones directas entre los promotores y otros agentes externos. La supervivencia y crecimiento de la CTA dependerá de su habilidad por mantener y extender sus redes. Sin embargo la utilización inadecuada o no utilización en cada una de las fases puede repercutir negativamente en su continuidad en el mercado.

En este trabajo hemos clasificado las redes en 5 grupos: Red privada, Red de Trabajo, Red Profesional, Red de Mercado, y Red Internacional; y utilizando una metodología cualitativa (Teoría Fundamentada) hemos identificado el tipo de red usado por los promotores en cada una de las fases, así como la repercusión de éstas en el éxito o fracaso respecto a la consolidación de la CTA.

La Teoría Fundamentada nos permite desarrollar una teoría substantiva que es significativa, compatible con el fenómeno observado y reproducible de forma rigurosa, que nos ayuda a explicar de forma exacta el fenómeno estudiado. A través del método de análisis comparativo constante, la saturación teórica, la clasificación teórica, el establecimiento de los procesos sociales básicos y la escritura teórica podemos definir con detalle lo que realmente está sucediendo.

Para el análisis se tomó como base de estudio la población total de CTA creadas durante los años 2006 y 2007 en la Comunidad Valenciana; seleccionando inicialmente 36 cooperativas siguiendo una proporción coherente con la distribución por sectores de actividad presentes en el conjunto de la muestra, y se realizó el estudio en el periodo que abarca de Enero a julio del 2013 aplicando la metodología indicada. Los resultados más concluyentes proporcionados por el análisis nos indicaron las redes que los nuevos emprendimientos cooperativos están utilizando durante su proceso de creación, así como el impacto que su uso 
tiene sobre el éxito o no en su consolidación. Los aspectos concluyentes en cada una de las etapas del proceso de creación de la CTA fueron los siguientes:

- Durante la primera fase del proceso (Acontecimientos Desencadenantes), la red privada es la que tiene un mayor peso en la decisión de crear una nueva CTA; y en segundo término la red de trabajo. No teniendo ninguna relevancia las demás redes del emprendedor.

- En la segunda fase del proceso (Concepto de Negocio) los emprendedores cooperativistas siguen apoyándose de forma significativa y equilibrada en las redes privadas y de trabajo. En este sentido, es importante resaltar que, aunque este apoyo les proporciona alto grado de confianza, las CTA que construyen fuertes relaciones de dependencia con estas redes ven limitada su supervivencia en el futuro. Las CTA más exitosas comienzan en esta fase a construir redes profesionales, que les proporcionan legitimidad en el mercado, mayor visibilidad y reputación.

- La tercera fase (Formalización de la empresa) se caracteriza por un uso escaso y poco significante, sobre todo en las CTA más exitosas, de las redes privadas y de trabajo; intensificando el protagonismo de la red profesional, que les proporciona una reputación que les permitirá establecer relaciones de confianza futura con proveedores y clientes en la siguiente fase.

- Finalmente, en la fase de Consolidación del negocio, se ha concluido que aquellas cooperativas que no mantenían redes de mercado o éstas eran débiles no habían sido capaces de mejorar sus rendimientos, y por consiguiente se encontraban actualmente en dificultades importantes de supervivencia. Sin embargo, la probabilidad de supervivencia no guardaba relación proporcional con el número de contactos mantenidos en el mercado; pues a partir de un número elevado de contactos (7-8 contactos) el rendimiento de la cooperativa se veía afectada. En esta etapa las redes internacionales son poco utilizadas, por una parte por el carácter local de estas cooperativas y por otra por su aversión al riesgo.

\section{BIBLIOGRAFÍA}

ALDRICH, H.E. (1999) Organizations Evolving. Sage: London.

BEL, P. y AUSIN, J.M. (2007) Contribución de las sociedades cooperativas al desarrollo territorial. REVESCO. Revista de Estudios Cooperativos, $\mathrm{n}^{\circ}$ 92, segundo cuatrimestre, pp. 41-71. 
CARRERO, V. (1998) Análisis Cualitativo de Datos: Aplicación de la Teoría Fundamentada (Grounded Theory) en el ámbito de la Innovación Organizacional. Tesis Doctoral Universidad Jaume I (Castellón).

CASSON, M. y DELLA, M. (2007) Entrepreneurship and social capital: Analysing the impact of social networks on entrepreneurial activity from a rational action perspective. International Small Business Journal, vol. 25, nº. 3, pp. 220-244.

CLINTON, M; TOTTERDEL, P. y WOOD, S. (2006) A Grounded Theory of Portafolio Working. International Small Business Journal, vol. 24, nº 2, pp. 179-203.

COLLINS, O.F. y MOORE, D.G. (1970) The Organitation makers. NewYork: AppletonCentury-Grofts.

CONNELL, J. y LOWE, A. (1997) Generating grounded theory from qualitative data: The application of inductive methods in tourism and hospitality management research. Progress in Tourism and Hospitality Research, vol. 3, pp. 165-173.

COYLE, I.T. (1997) Sampling in qualitative research: purposeful and theoretical sampling; merging or clear boundaries?. Journal of Advanced Nursing, vol. 26, pp. 623-630.

CUÑAT, R. J. (2005) La realidad de las nuevas Cooperativas de Trabajo Asociado: de la idea a la consolidación. Sociedad Cooperativa. Noviembre 2005, pp. 23-26.

CUÑAT, R. J. y COLL, V. (2006) Análisis de los factores que influyen en el proceso de creación de una cooperativa de trabajo asociado. REVESCO. Revista de Estudios Cooperativos, $\mathrm{n}^{\circ} 88,1^{\circ}$ Cuatrimestre de 2006, pp. 128-161.

DOUGLAS, D. (2004) Grounded Theory and the "And" in Entrepreneurship Research. Electronic Journal of Business Research Methods, vol. 2, pp. 59-68.

ELFRING, T. y HULSINK, W. (2003) Networks in entrepreneurship: The case of hightechnology firms. Small Business Economic, vol. 21, nº. 4. pp. 409-422.

ETEMAD, H. (2004) International Entrepreneurship as a Dynamic Adaptative System: Towards a Grounded Theory. Journal of International Entrepreneurship, vol. 2, pp. 5-59.

FIELDING, N.G. y LEE, R.M. (1998) Computer analysis and qualitative research. New technologies for social research. London: Sage.

GARCÍA-GUTIÉRREZ, C. (1991) La Economía social o la economía de las empresas de participación (las sociedades cooperativas laborales) en: Memoria de Maria Ángeles Gil Luezas. Alfa Centauro, Madrid, pp. 195-216.

GIBSON, B., GREGORY, J. y ROBINSON, P. (2005) The intersection between theory and grounded theory: The emergence of the grounded system observer. Qualitative Sociology Review, vol. I, pp. 3-21. 
GLASER, B.G. (1992) Basics of grounded theory analysis: Emerge vs. Forcing. Mill Valley, CA: Sociology Press, California.

GLASER, B.G. (2004) Naturalist Inquiry and Grounded Theory. Forum: Qualitive Social Research 5 (2). Retrieved November 30, 2005 (http://www.qualitative-research.net/fqstexte/1-04/1-04glaser-e.pdf )

GLASER, B.G. (1978) Theoretical Sensitivity: Advances in the Methology of Grounded Theory. Mill Valley, CA: Sociology Press, California.

GLASER, B.G. (2002) Constructivist Grounded Theory?. Forum: Qualitative Social Research 3 (3). Retrieved November 30, 2005 (http: www.qualitative-research.net/fqstexte/3-02/3-02glaser-e.htm )

GLASER, B.G. y STRAUSS, A.L. (1967) The Discovery of Grounded Theory: strategies for qualitative research. Aldine, New York.

GREVE, A. y SALAFF, J.W. (2003) Social networks and entrepreneurship. Entrepreneurship theory and practice, 28(1), pp. 1-22.

HENWOOD, K. y PIDGEON, N. (1995) Using Grounded Theory in Psychological Research. Lawrence Erlbaum, USA.

HERRERA, H. (2009) Investigación sobre redes socials y emprendimiento: revisión de la literatura y agenda futura. Innovar, vol. 19, n 33, pp. 19-33.

HOANG, H. y ANTOCIC, B. (2003) Network-based research in entrepreneurship: a critical review. Journal of Business Venturing, vol. 18, pp. 165-187.

JENSSEN, J.I. y KOEING, H.F. (2002) The effect of social networks on resource access and business start-ups. European Planning Studies, Vol. 10, pp. 1039-1046.

JEZEWSKI, M.A. (1995) Evolution of a grounded theory: conflict resolution through culture brokering. Advances in Nursing Science, vol. 17, pp. 14-30.

JOHANNISSON, B. y MONSTED, M. (1997) Contextualizaing entrepreneurial networking. International Studies of Management and Organization, vol. 27, nº 3, pp.109-136.

KNOKE, D. y BURT, R. (1986) Prominence. in Burt, Ronald S., and Michael j. Minor (eds). Applied Network Analysis: A methodological Introduction. Beverly Hills: Sage, 195-222.

LECHNER, C. y DOWLING, M. (2003) Firm networks: External relationship as sources for the growth and competitiveness of entrepreneurial firms, Entrepreneurship and Regional Development, 15, pp. 1-26.

LECHNER, C., DOWLING, M. y WELPE, I. (2006) Firm networks and firm development: The role of the relational mix. Journal of Business Venturing, vol. 21, pp. 514-540. 
LEJARRIAGA, G., BEL, P. y MARTIN, S. (2013) El emprendimiento colectivo como salida laboral de los jóvenes: Análisis del caso de las Empresas Cooperativas de Trabajo Asociado. REVESCO. Revista de Estudios Cooperativos, n 112, pp. 36-65.

LOWE, A. (1995) The basic social processes of entrepreneurial innovation. International Journal of Entrepreneurial Behaviour and Research, vol. 2, pp. 54-55.

MADSEN, E.L. (2007) The significance of sustained entrepreneurial orientation of perfomance of firms- A longitudinal analysis. Entrepreneurship \& Regional Development, vol. 19, pp. 185-204.

MARTIN, S. y LEJARRIAGA, G. (2011) Las empresas de participación de trabajo asociado ante los desafíos del futuro en un contexto de crisis económica: propuesta de actuación. CIRIEC-ESPAÑA, no 72, Octubre 2011, pp. 239-261.

MONZÓN, J. L. y MORALES, A.C. (1998) Competencias y valores en las empresas de trabajo asociado. CIRIEC-ESPAÑA, Valencia.

MORALES, A.C.; ARIZA, J.A. y MUÑIZ, N.M. (2012) El emprendedor social y el Eempowerment de las redes virtuales. CIRIEC-ESPAÑA, vol. 75, pp. 153-177.

ORELLANA, W. y MARTÍNEZ DE LEJARZA, J. (2013) Teorías de entrepreneurship y cooperativismo de trabajo asociado. Fundamentos teóricos y evidencias empíricas en la creación de CTA, CIRIEC-ESPAÑA, n 78, pp. 11-36.

PARTINGTON, D. (2000) Building grounded theories of management action. British Journal of Management, vol. 11, pp. 91-102.

PUENTES, R. y VELASCO, M.M. (2009) Importancia de las Sociedades Cooperativas como medio para contribuir al desarrollo económico, social y medioambiental, de forma sostenible y responsable. REVESCO. Revista de Estudios Cooperativos, $\mathrm{n}^{\circ}$ 99, Tercer cuatrimestre 2009, pp. 104-129.

RODRIGO, B. (1995) La excelencia empresarial en la sociedad cooperative. CIRIECESPAÑA, no 19 extraordinario, octubre 1995, pp. 43-53.

SALAFF, J.W. (2003) Social Networks and Entrepreneurship, Entrepreneurship, Theory \& Practice, vol. 28, no. 1, pp. 1-22.

SANCHIS, J.R. (2001) Creación y consolidación de empresas mediante crecimiento en red: su aplicación al desarrollo local. Dirección y Organización, no 25, pp. 14-25.

SCHOTT, T. y CHERAGHI, M. (2012) Entrepreneurs'networks: Size, diversity and composition shaped by cultures of rationality and trust. IEEE Computer Society, vol.2, pp. $220-226$. 
SOLYMOSSY, E. (2000) Knowledge networks: differences and performance effects. Journal of Business Strategy, vol. 11, n. 1, pp. 14-25.

SPIEGELBERG, H. (1994) Analysis and interpretation of qualitative data in consumer research. Journal of Consumer Research, vol. 21, pp. 491-503.

STRAUSS, A.L. (1987) Qualitative analisis for social scientifics. Cambridge University Press, New York.

STRAUSS, A.L. y CORBIN, J. (1990) Basics of Qualitative Research: Grounded Theory, procedures and techniques. Sage Publications, Newbury Park, CA.

TIMMONS, J.A. (1990) New Venture Creation: Entrepreneurship in the 1990's. Ed. Irwin Homewood, Irwin.

VALLES (2005) Metodología y Tecnología cualitativas: Actualización de un debate, desde la mirada atenta en la obra de Barney G. Glaser. Empiria, nº 9, pp. 145-170.

VENKATARAMAN, S. y VAN DE VEN, A. (1998) Hostile environmental jolts, transaction set, and new business. Journal of Business Venturing, vol. 13, pp. 231-255.

VESPER, K.H. (1990) New Venture Strategies. Ed. Prentice-Hall, Englewood Cliffs, N.J.

ZANDONAL, F. y PEZZINI, E. (2004) Nuevos directivos para nuevas cooperativas. La figura del empresario social en las cooperativas sociales italianas. CIRIEC-ESPAÑA, $\mathrm{n}^{\circ}$ 48, pp. 145-165. 